\title{
Bar Properties as a Function of Hubble Type
}

\author{
Debra Meloy Elmegreen \\ Vassar College, Poughkeepsie, NY 12601
}

\begin{abstract}
Previous optical surface photometry of barred spiral galaxies revealed that there are two distinct types of bars: large bars tend to have a nearly constant surface brightness ("flat" bar), while smaller bars tend to have a decreasing surface brightness with a scale length similar to the disk ("exponential" bar). Statistically, flat bars tend to occur in early Hubble types and exponential bars in later types. Studies of resonances in spirals indicate that flat bars end inside corotation, while exponential bars end between the inner Lindblad and 4:1 resonances. Near-infrared $(J H K)$ surface photometry of bars is presented in order to compare the stellar distributions and bar potentials in flat and exponential barred galaxies. The presence of isophotal twists in some galaxies provides additional information on resonances. The grand design and flocculent optical structures in the two types of barred galaxies will be compared and contrasted with their near-infrared light distributions.
\end{abstract}

\section{Bar Size and Type versus Hubble Type}

The morphology of spirals and bars in barred galaxies can be quite varied in different Hubble types. Near-infrared images of galaxies like NGC 7479 and NGC 1300 show very strong bars with two long symmetric arms originating at the ends of the bars. In contrast, galaxies like NGC 2500 and NGC 1359 show small weak central bars in disks with very little spiral structure of any kind. Previously, we showed that the bars in early Hubble types (taken here to be $\mathrm{SBa}-\mathrm{SBbc}$ ) have $B$ and $I$ light profiles which are approximately constant along the bars ("flat" bars; Elmegreen \& Elmegreen 1985). Galaxies in later Hubble types (SBc-SBm) have light profiles which decrease with a scale length virtually indistinguishable from that of the disk ("exponential" bars). Our recent $J H K$ band observations of a dozen early and late barred galaxies confirm the previous results; less extinction and non-saturated central regions make the identification of flat and exponential bars more certain (Elmegreen et al. 1995).

Figure 1 shows radial profiles along and perpendicular to the bars of the early type galaxy NGC 1097 and the late type galaxy NGC 1359 in the $J$ band. The bars in early types extend well beyond the bulge region and are very flat. Occasionally, as in NGC 1300, star formation is enhanced at bar's end, so the young supergiants in these regions contribute some to the $J$ band and the bars show small bumps at their end. The late type bars, in contrast, are difficult to distinguish from the disk and bulge, and all components connect smoothly. 


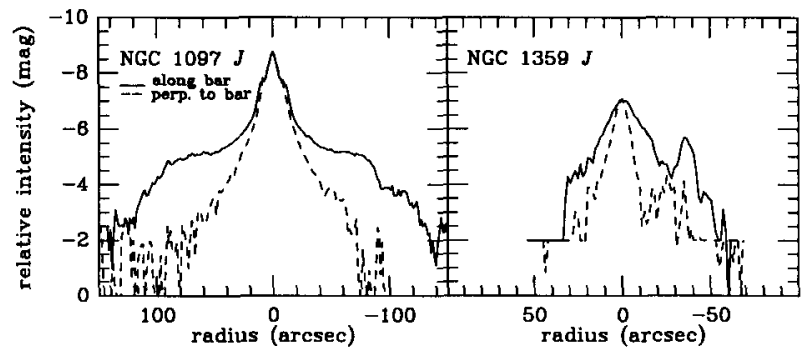

Figure 1. Radial profiles are shown along the bar (solid) and perpendicular to the bar (dashed) in NGC 1097 and NGC 1359.

The relative inclination-corrected bar size varies as a function of galaxy type; flat bars are typically 0.3-0.6 $R_{25}$ and exist in early-type galaxies, while exponential bars range from $0.1-0.3 R_{25}$ and exist in late-type galaxies (Elmegreen \& Elmegreen 1985 and this study, and Martin 1995). The corrected relative bar length is independent of absolute blue magnitude, except for the dichotomy between the brightnesses of early and late-type spirals. Our previous study also showed that the length of the bar tends to extend beyond the rising part of the rotation curve in flat barred galaxies, but inside the rising part in exponential barred galaxies. The corrected bar axial ratios were studied by Martin (1995), who found a wide range between 0.2 and 0.9 that is independent of Hubble type.

Most galaxies have outer arms that extend near $\mathrm{R}_{25}$, with two inner symmetric arms that have a break or noticeable decrease in brightness partway out in the disk. The inclination-corrected endpoints of the inner symmetric arms correlate with the relative length of the bar, regardless of the bar type (Elmegreen \& Elmegreen 1995). In order to compare the symmetry and bar ends with resonances, we assumed that the rotation curves have power law fits (Elmegreen, Elmegreen, \& Montenegro 1991) and that the slope of the curve depends on galaxy absolute magnitude (Persic \& Salucci 1990). Most spiral arms end within about $10 \%$ of $R_{25}$ (EEM); if we identify this end with the outer Lindblad resonance (OLR), we can determine the locations of other resonances in the disks based on the slope of the rotation curve. The bars in early-type galaxies end somewhere between the inner 4:1 resonance and the corotation resonance (Elmegreen \& Elmegreen 1995), in good agreement with orbit theory (Combes \& Gerin 1985, Patsis et al. 1991). In late type galaxies, the inner two arms end near corotation, and the bars end between the 4:1 resonance and the inner Lindblad resonance.

\section{Interactions versus Hubble Type}

A statistical study of the distributions of galaxies in different environments was made for early, intermediate, and late spiral Hubble types (Elmegreen, Elmegreen \& Bellin 1990). We determined the fraction of spirals that had bars among all spirals in field galaxies, in group galaxies, and in binaries. The frac- 
tion in intermediate types was only $20-30 \%$ regardless of environment, while the fraction in late types was $60-90 \%$ in all environments. On the other hand, among early Hubble types, the fraction of barred galaxies was about twice as high in binaries $(50 \%)$ as in field or group galaxies (25\%), suggesting a strong link between close interactions and flat bars. Statistically, there was an excess of early barred Hubble types and a deficit of intermediate ovally distorted and barred Hubble types in binary systems relative to field galaxies. This result suggests that interactions simultaneously make bars and bigger bulges. Giuricin et al. (1993) examined the cumulative function for different types of galaxies as a function of different average spacings to nearest neighbors. They also found that the only difference between barred and nonbarred galaxies was among early Hubble types, in which the barred galaxies were found to be in regions of higher density than nonbarred spirals.

\section{Bars versus Arm Class}

The distributions of different Hubble and bar types were also examined with respect to the arm class of a galaxy, which is a measure of the overall arm symmetry and continuity (cf. Elmegreen \& Elmegreen 1987). The presence of grand design structure ( 2 main arms) or multiple arm structure with inner twoarm symmetry signifies the presence of a spiral density wave. The fraction of intermediate Hubble type spirals having a density wave was $60-70 \%$, while the fraction for late Hubble type spirals was only $5 \%$, regardless of the presence or absence of a bar. In contrast, the fraction for early Hubble types was $80 \%$ for barred galaxies and only $45 \%$ for nonbarred galaxies. Thus, only flat bars are correlated with spiral arm structure.

\section{Bar-Interbar Contrasts}

The arm-interarm contrast, which is the difference in magnitude between an arm and its interarm region at the same radius, was found to increase with radius in $B$ and $I$ band for nonbarred and exponential barred galaxies but to decrease or remain constant beyond the bar for flat barred galaxies (Elmegreen \& Elmegreen 1985). We have determined the $J$ and $K$ band bar-interbar and arm-interarm contrast for several barred galaxies (Elmegreen et al. 1995). Consistent with the $I$ band results, the bar-interbar contrast increases with radius for all galaxies. Perhaps surprisingly, the bar-interbar contrast has the same range of magnitude differences (1-3) and the same slope for both flat and exponential bars. The contrast continues to increase beyond the bar length for exponential bars, but declines or levels beyond the bar for flat bars. For exponential bars, a decline or levelling occurs about halfway out in the disk. In all cases, these declines are in the vicinity of corotation, which is consistent with ideas that density waves are reinforced only out to corotation (Lin \& Lowe 1990). 


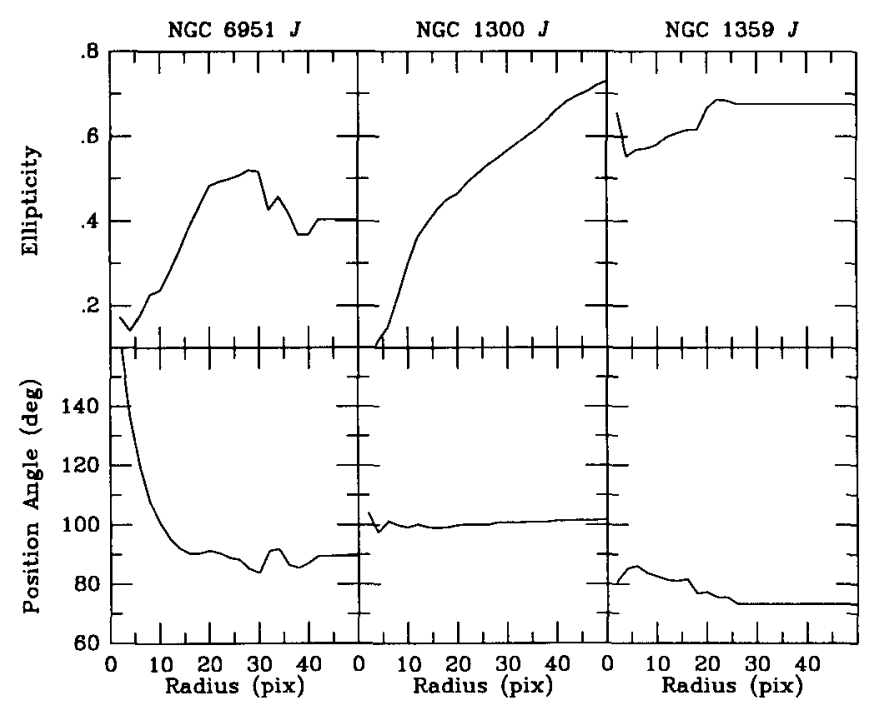

Figure 2. Position angles (solid lines) and ellipticities (dashed lines) are shown as a function of radius $(1$ pix $=1 ! 4)$ for three galaxies.

\section{Isophotal Twists}

The $J H K$ contours for early type barred galaxies often, but not always, show the presence of isophotal twists. Previous studies have reported twists in about a dozen galaxies (Kormendy 1983, Jarvis et al. 1988, Buta \& Crocker 1993, Shaw et al. 1993), and Wozniak et al. (1995) report about two dozen more. We also note that the contours of interacting Arp galaxies published by Bushouse and Stanford (1992) show about 7 galaxies with twists, of which 2 galaxies are known to be early type barred galaxies (the others are not classified). In this study (Elmegreen et al. 1995), there are 16 barred galaxies studied in $I J H K$ bands: 9 flat, with 5 having twists, and 7 exponential, with none having twists.

Ellipse fits are shown in Figure 2 for three galaxies: NGC 6951 is a flat barred galaxy with a twist from $160^{\circ}$ in the center to $90^{\circ}$ at the bar; the position angle then remains constant in the rest of the disk. The ellipticity for this galaxy reaches its maximum at the main bar. NGC 1300 is a flat barred galaxy with no twist; its position angle is a constant $105^{\circ}$ till the spiral arms begin, and the ellipticity is a maximum of $70 \%$ for the bar. NGC 1359 is an exponential barred galaxy with no twist. Its position angle is $86^{\circ}$ in the barred region and declines smoothly in the disk, and its ellipticity only varies a few percent. There are many possible interpretations for isophotal twists, depending on the individual galaxy (cf. Friedli, these proceedings). Twists mostly likely represent bars within bars, in which case they are purely stellar and co-exist with two different pattern speeds (e.g., Friedli \& Martinet 1993, Wozniak et al. 1995). In some galaxies, the twists could be due to an ILR where the gas is collected inside the ILR. 
The gas response is offset in angle to the bar, and subsequent star formation or star alignment in the offset gas would show a twist (e.g. Shaw et al. 1993). On the other hand, in some cases the twists could just be projection effects. The position angles of the inner twists in this study are random with respect to the main bars and outer disks, suggesting that these are not just projection effects.

\section{Summary}

We conclude that, in early type galaxies, the bars have flat light profiles and are long, ending between corotation and the inner 4:1 resonance. Some of the bars show inner isophotal twists. The bars are associated with grand design or multiple arm spiral structure, and statistics show that some of the bars are the result of interactions. In contrast, late type galaxies have bars with exponential light profiles. The short bars end before $0.3 R_{25}$, between ILR and the $4: 1$ resonance. These bars do not determine the arm structure, nor does environment appear to play a role in their existence. No twists are seen. For all Hubble types, the bars have the same wide range in axial ratio and the same bar-interbar contrast which increases with radius to at least the end of the bar. The inner two arm structures end at about twice the bar radius.

\section{References}

Bushouse, H. A. \& Stanford, S. A. 1992, ApJS, 79, 213

Buta, R. \& Crocker, D. 1993, AJ, 105, 1344

Combes, F. \& Gerin, M. 1985, A\&A, 150, 327

Elmegreen, B. G. \& Elmegreen, D. M. 1989, ApJ, 342, 677

Elmegreen, B. G. \& Elmegreen, D. M. 1985, ApJ, 288, 438

Elmegreen, B. G., Elmegreen, D. M., \& Montenegro, L. 1992, ApJS, 79, 37

Elmegreen, D. M. \& Elmegreen, B. G. 1987, ApJ, 314, 3

Elmegreen, D. M. \& Elmegreen, B. G. 1995, ApJ, 445, 541

Elmegreen, D. M., Elmegreen, B. G., \& Bellin, A. 1990, 364, 415

Elmegreen, D. M., Elmegreen, B. G., Chromey, F. R., Hasselbacher, D. A., \& Bissell, B. 1995, ApJ, submitted

Friedli, D. \& Martinet, L. 1993, A\&A, 277, 27

Giuricin, G., Mardirossian, F., Mezzetti, M., \& Monaco, P. 1993, ApJ, 407, 22 Jarvis, B. J., Dubath, P., Martinet, L., \& Bacon, R. 1988, A\&AS, 74, 513

Kormendy, J. 1983, ApJ 275, 529

Lin, C. C. \& Lowe, S. A. 1990, in Galactic Models, J. R. Buchler, S. T. Gottesman \& J. H. Hunter, Ann. N. Y. Acad. Sci. Vol. 596, 80

Martin, P. 1995, AJ, 109, 2428

Patsis, P. A., Contopoulos, G., \& Grosbol, P. 1991, A\&A, 243, 373

Persic, M. \& Salucci, P. 1991, ApJ, 368, L60

Quillen, A. C., Frogel, J. A., \& Gonzalez, R. A. 1994, ApJ, 437, 162

Shaw, M. A., Combes, F., Axon, D. J., \& Wright, G. S. 1993, A\&A, 273, 31 
Wozniak, H., Friedli, D., Martinet, L., Martin, P., \& Bratschi, P. 1995, A\&AS, 111,115

\section{Discussion}

A. Zasov: You combined SAB and SB galaxies. Do SAB galaxies have intermediate properties or are they closely related to SB ones?

D. Elmegreen: We examined SAB galaxies separately from SB galaxies. However, in all cases their distributions and photometric properties were identical, so I grouped them together here.

D. Pfenniger: Because the overall light profile in early-type galaxies is quite steep when the bulge light is included, I guess that the flat bar characteristics you describe may be strongly dependent on the bulge model you subtract. Dynamically there is no justification for a particular bulge model, especially for a spherical one.

D. Elmegreen: I did not subtract bulge from the profiles I showed. The bulge component is quite obvious in the central regions, and distinct (clearly resolved) from the bar in its outer parts.

J. Palous: Could the flatness of some bars be connected with secondary star formation maxima combined with limited angular resolution?

D. Elmegreen: We do see star formation peaks at the ends of some early type bars, which show a rise in the light profile in $B$-band. These contributions are minimized by going to $J H K$ bands. The flat bars are still evident even in the parts of the bars without obvious star formation.

D. Friedli: Do galaxies with a flat bar have a lens? Could the shoulder in the density profile simply be the signature of a lens?

D. Elmegreen: Most of these early type galaxies do not have lenses. Those that do have bars which fill the lenses.

$F$. Combes: Are there some rings in your sample of galaxies, and do you see the characteristic bump in the radial profile? (Some inner rings could be the origin of the flat profile).

D. Elmegreen: No, none of these galaxies has an inner ring. One has an outer ring, and that of course shows up in the profile.

$K$. Freeman: The N-body bars that arise in disk simulations: are they like your flat bars, or more like the exponential ones?

D. Elmegreen: Generally they are like the flat bars, although not everyone doing models plots the density distribution. Elmegreen \& Combes have a model which can reproduce the exponential bars (see B. Elmegreen, these proceedings).

$H$. Ann: I have examined about 100 barred galaxies, and find that all the galaxies with isophotal twists are early type or late type galaxies. Do you know why there are no intermediate type galaxies with isophotal twists? 
D. Elmegreen: I'm surprised to learn that there are any late type galaxies with isophotal twists! It seems that in many cases the twists depend on an ILR or bulge, both of which are largely absent in later types.

A. Quillen: Does the radial variation of ellipticity in bars depend upon bar type (exponential versus flat)? One might expect flat bars to have varying ellipticity along the bar and exponential bars to have constant ellipticity. This would relate to the extent and shape of $x_{1}$ orbits. Exponential bars probably rarely have ILRs. Since azimuthal averages are exponential disks (Ohta, these proceedings), the division into flat and exponential could just be because of the existence or not of an ILR, which is the same as the existence or not of a bulge. This would explain the correlation with Hubble type.

D. Elmegreen: Yes, there is a variation in ellipticity with Hubble type, and your explanation sounds sensible. 